\title{
Expression of Birt-Hogg-Dubé gene mRNA in normal and neoplastic human tissues
}

\author{
Michelle B Warren ${ }^{1}$, Carlos A Torres-Cabala ${ }^{2}$, Maria L Turner ${ }^{3}$, Maria J Merino², \\ Vera Y Matrosova ${ }^{4}$, Michael L Nickerson ${ }^{1}$, Wenbin $\mathrm{Ma}^{1}$, W Marston Linehan ${ }^{5}$, \\ Berton Zbar ${ }^{1}$ and Laura S Schmidt ${ }^{6}$ \\ ${ }^{1}$ Laboratory of Immunobiology, Center for Cancer Research, NCI Frederick, Frederick, MD, USA; ${ }^{2}$ Laboratory \\ of Pathology; ${ }^{3}$ Dermatology Branch, Center for Cancer Research, NCI, NIH, Bethesda, MD, USA; ${ }^{4}$ Department \\ of Pathology, Uniformed Services University of the Health Sciences, Bethesda, MD, USA; ${ }^{5}$ Urologic Oncology \\ Branch, Center for Cancer Research, NCI, NIH, Bethesda, MD, USA and ${ }^{6}$ Basic Research Program, SAIC- \\ Frederick, Inc., NCI Frederick, Frederick, MD, USA
}

\begin{abstract}
Birt-Hogg-Dubé (BHD) syndrome is an inherited autosomal genodermatosis characterized by hamartomas of the hair follicle called fibrofolliculomas and an increased risk for developing spontaneous pneumothorax, lung cysts and renal neoplasia. BHD was localized to chromosome 17p11.2 by linkage analysis in BHD families, and germline insertion/deletion and nonsense mutations in a novel gene were identified which were predicted to prematurely truncate the BHD protein, folliculin. No homology to other human proteins was found although folliculin was conserved across species. As a first step toward understanding the function of $B H D$ in the cell and how BHD mutations can lead to the BHD phenotype, we measured the expression of BHD mRNA in normal and neoplastic human tissues by fluorescent in situ hybridization. BHD mRNA was expressed in a variety of tissues, including the skin and its appendages, the distal nephron of the kidney, stromal cells and type 1 pneumocytes of the lung, acinar cells of the pancreas and parotid gland, and epithelial ducts of the breast and prostate. In the brain, BHD mRNA was expressed in neurons of the cerebrum, and Purkinje cells in the cerebellum. BHD mRNA was also expressed in macrophage and lymphocytes in the tonsils and spleen. Tissues with reduced expression of $B H D$ mRNA included heart, muscle and liver. BHD mRNA was expressed strongly in the proliferating epithelial strands of fibrofolliculomas, the cutaneous lesions characteristic of BHD, but not in renal tumors from BHD patients. These results indicate a wide expression pattern for BHD mRNA in many tissues, including skin, lung and kidney, which are involved in the BHD phenotype, and support a tumor suppressor role for $B H D$ in renal cancer.
\end{abstract}

Modern Pathology (2004) 17, 998-1011, advance online publication, 14 May 2004; doi:10.1038/modpathol.3800152

Keywords: Birt-Hogg-Dubé syndrome; BHD gene; renal carcinoma; tumor suppressor gene; fluorescent in situ hybridization; mRNA expression

Birt-Hogg-Dubé (BHD) syndrome is a genodermatosis inherited as an autosomal dominant trait. The disorder, first recognized in $1977,{ }^{1}$ is characterized by the presence of multiple skin papules on the head and neck accompanied by acrochordons (skin tags) and trichodiscomas. Histologically, the cutaneous papules consist of thin, epithelial strands originating from a central hair follicle with prominent associated connective tissue. These skin lesions were named fibrofolliculomas and considered

Correspondence: Dr LS Schmidt, PhD, Laboratory of Immunobiology, National Cancer Institute Frederick, Bldg 560, Rm 12-69, Frederick, MD 21702, USA.

E-mail: schmidtl@mail.ncifcrf.gov

Received 23 December 2003; revised 15 March 2004; accepted 23 March 2004; published online 14 May 2004 to be hamartomas of the hair follicle. Since the original description of the disorder by Drs Birt, Hogg and Dubé, BHD families with characteristic dermatologic lesions and other phenotypic features have been reported including renal neoplasia, ${ }^{2}$ lung cysts, and/or spontaneous pneumothorax. ${ }^{3,4}$ Patients with fibrofolliculomas have a 50-fold increased risk for developing spontaneous pneumothorax, and a seven-fold increased risk for renal carcinoma. ${ }^{5}$ Patients affected with BHD syndrome are predisposed to develop several different histologic types of renal carcinoma including clear cell, papillary and chromophobe renal carcinomas, renal oncocytomas and a hybrid tumor containing elements of both chromophobe renal carcinoma and renal oncocytoma. ${ }^{6,7}$ The oncocytic hybrid renal tumor is the most common renal neoplasm found in BHD patients. 
The human $B H D$ gene was localized to chromosome $17 \mathrm{p} 11.2$ by linkage analysis ${ }^{8,9}$ and identified by positional cloning. ${ }^{10}$ Nearly all $B H D$ gene mutations identified in the germ line of BHD patients are predicted to result in frameshifts, which produce a truncated protein. Almost half of BHD patients harbor an insertion/deletion mutation in a tract of eight cytosines leading to premature protein termination. The full-length $B H D$ sequence encodes a novel protein, folliculin, with an open reading frame of 579 amino acids and no significant homology to known human proteins by BLAST alignment against SwissPro and nonredundant NCBI protein databases. The amino-acid sequence of folliculin predicts a $64 \mathrm{kDa}$ protein with a short hydrophobic $\mathrm{N}$-terminal sequence, one N-glycosylation site, three myristoylation sites and a glutamic acid-rich coiledcoil domain centrally located in the BHD protein. No transmembrane domains or organelle localization signals were identified by computer-assisted searches (Expert Protein Analysis System proteomics server of the Swiss Institute of Bioinformatics at http://us.expasy.org). Homologs of the BHD protein have been identified in the mouse, Drosophila and Caenorhabditis elegans. Interestingly, two inherited forms of renal cancer in the dog and the rat may be caused by mutations in homologs of human $B H D$. A naturally occurring form of inherited renal cancer and nodular dermatofibromatosis in the German Shepherd $\operatorname{dog}^{11,12}$ has been shown to cosegregate with a missense mutation in the dog $B H D$ gene; ${ }^{13}$ the Nihon rat cancer model, a spontaneously occurring inherited renal cancer in the Sprague-Dawley rat, has been linked to a region of the rat chromosome 10 which is syntenic with human chromosome 17p11.2. ${ }^{14,15}$ Homozygosity of the $B H D$ homolog alleles in rat and dog is embryonic lethal.

By Northern blot analysis a $3.8 \mathrm{~kb} B H D$ transcript was detected in a wide variety of adult tissues, including brain, heart, placenta, testis, skin, lung and kidney, as well as fetal lung, liver, brain and kidney. ${ }^{10}$ As a first step toward understanding the biological function of this novel renal cancerassociated gene, we evaluated the specific cell type and tissue distribution of $B H D$ mRNA by fluorescent in situ hybridization in normal and neoplastic human tissues, and selected abnormal and neoplastic tissues from BHD patients.

\section{Materials and methods}

\section{Tissue Specimens}

Both fresh frozen tissue and paraffin-embedded tissue were used in this study. The in situ hybridization protocol was optimized by hybridizing probe to fresh frozen and paraffin-embedded human scalp samples (voluntarily obtained through the NIH Dermatology Service at the NIH Clinical Center with patient informed consent). Paraffin-embedded nor- mal and neoplastic tissue was obtained from the archives of the NIH Laboratory of Pathology.

Two tissue arrays were used to study the expression of $B H D$ mRNA in normal and neoplastic tissues. A low-density Landmark Tissue MicroArray (TMA) was obtained from Ambion with 22 normal tissues each represented by samples from four individuals. For comparison of normal tissue expression to neoplastic tissue, a low-density Tissue MicroArray of 34 cancerous tissue samples (Ambion) with matched benign specimens was also hybridized. To specifically examine renal carcinoma samples, a custom tissue array was produced by the Tissue Array Research and Production Lab of the National Cancer Institute with samples of oncocytoma, oncocytic hybrid, papillary and clear cell histologic types obtained from patients with BHD, hereditary papillary renal carcinoma or von HippelLindau disease.

\section{In Situ Hybridization Probes}

In situ hybridization was performed using a riboprobe designed from the cDNA sequence of the $B H D$ gene. The primers for the $B H D$ probe were designed and synthesized from exons 11, 12 and 13 of the $B H D$ mRNA sequence ( $\mathrm{F}$ 5'-CTG TGT TGC CAG AGA GTA CAG AAG G-3' ${ }^{\prime}$ and R 5'-CCA CAG ACA GGT TCT GGT TGG TC-3'). The primers were used to PCR amplify a 561 bp product from $B H D$ cDNA isolated from fetal heart (Clonetech) using standard conditions with a $64^{\circ} \mathrm{C}$ annealing temperature. Sp6 adapter ends containing a primer sequence were ligated directly to the PCR product using the Lig-nScribe kit (Ambion) following the manufacturer's protocol, and PCR-amplified to produce sense and antisense templates. The templates were in vitro transcribed with fluorescein-labeled dUTP (Roche) using the MAXIscript in vitro transcription kit (Ambion) and treated with DNase I per manufacturer's protocol.

A riboprobe to a keratin known to be expressed in the inner root sheath of the hair follicle, Ker6IRS $1{ }^{16}$ was used as a positive control and to optimize the in situ hybridization method. PCR primers for Ker6IRS1 were designed (F 5'-CAT CAG CAG CAC CAG TGG CGG-3' and R 5'-CAG GAA CTA TGC TAG GTC CCA $\mathrm{G}-3^{\prime}$ ) and the probe of interest was amplified by PCR at a $64^{\circ} \mathrm{C}$ annealing temperature using standard conditions. Ker6IRS1 was designed as a 589 bp PCR probe amplified from the $3^{\prime}$ UTR of the KER6 gene using genomic DNA as the template.

Tissue Preparation, Pretreatment, Hybridization and Signal Detection

The tissue samples were flash frozen in OCT media on dry ice and then sectioned using a cryostat at a thickness of 8-10 $\mu \mathrm{m}$. Paraffin sections $(5 \mu \mathrm{m})$ were deparaffinized by soaking in xylene and rehydrated 
in gradient alcohol washes. Frozen sections and dewaxed paraffin sections on slides were fixed at room temperature in $4 \%$ paraformaldehyde prepared in phosphate-buffered saline. Pretreatment of slides included quenching endogenous peroxidase with $1-3 \%$ hydrogen peroxide for $10 \mathrm{~min}, 25 \mu \mathrm{g} / \mathrm{ml}$ proteinase $\mathrm{K}$ for $7 \mathrm{~min}$ and an acetic anhydride treatment in $0.1 \mathrm{M}$ triethanolamine with $25 \mathrm{mM}$ acetic anhydride (added right before slide addition) for $10 \mathrm{~min}$. The slides were incubated with prehybridization solution containing $0.3 \mathrm{M} \mathrm{NaCl}, 20 \mathrm{mM}$ Tris pH 7.5, 5 mM EDTA, $1 \times$ Denhardt's solution, $0.5 \mathrm{mg} / \mathrm{ml}$ yeast tRNA, and $60 \%$ formamide for $2 \mathrm{~h}$ at $37^{\circ} \mathrm{C}$. Hybridization solution was prepared same as the prehybridization solution with the addition of $10 \%$ dextran sulfate. The hybridization solution was heated to $70^{\circ} \mathrm{C}$, and then probe $(1 \mathrm{ng} / \mu \mathrm{l}$ final concentration) was added and heated for an additional $5 \mathrm{~min}$. The probe and hybridization solution were cooled on ice, applied to the slides, and incubated overnight at $37^{\circ} \mathrm{C}$. Serial tissue sections were probed with the antisense and sense probes separately, and a third section was stained with hematoxylin and eosin to visualize tissue morphology.

Following hybridization, the slides were washed in $1 \times$ SSC and subjected to RNase A treatment $(0.131-1.0 \mu \mathrm{g} / \mathrm{ml}$ in PBS, Ambion) for $10 \mathrm{~min}$ at $37^{\circ} \mathrm{C}$. Slides were soaked twice for $8 \mathrm{~min}$ in a $50^{\circ} \mathrm{C}$ solution of $50 \%$ formamide in $2 \times$ SSC. Next, slides were run through a series of SSC-containing washes $(1 \times, 0.1 \times, 4 \times / 0.1 \%$ Tween 20$)$ at $50^{\circ} \mathrm{C}$ while shaking. The hybridized signal was detected using the Tyramide System Amplification (TSA) Plus kit for fluorescein from Ambion using the manufacturer's suggested protocol. Mounting media containing DAPI nuclei stain (Vector Laboratories) was added to the slides and slides were examined using a Zeis Axioplan epifluorescence microscope with DAPI and FITC filters from Chroma. The slides were photographed using a black and white Photometrics Cool Snap camera and IP-Lab 3.6.3-imaging software was used to layer and pseudocolor the images.

\section{Results}

\section{Optimization of the In Situ Hybridization Protocol}

Our initial experiments utilized standard published fluorescent in situ hybridization methods, but did not produce satisfactory results. Therefore, modifications to the basic procedure were necessary to optimize the in situ hybridization protocol (Figure 1). We found that paraffin-embedded tissue sections provided less background signal than frozen sections and better tissue morphology. Proteinase K was used at the highest concentration possible which did not damage the tissue section. Addition of dextran sulfate to the hybridization buffer produced a uniform signal and the increased viscosity helped prevent the slide from drying during the overnight

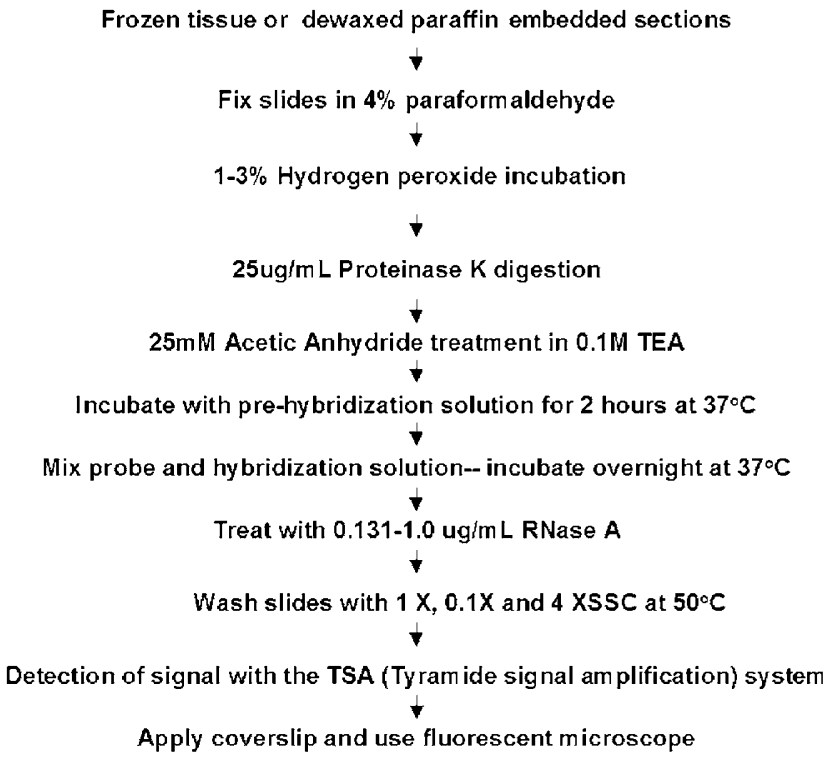

Figure 1 Flowchart of the optimized in situ hybridization protocol.

incubation. Higher hybridization temperatures damaged the tissue sample; it was found that a lower hybridization temperature of $37^{\circ} \mathrm{C}$ preserved tissue integrity, and increased wash stringency helped to reduce background. Pretreatment with acetic anhydride and treatment of the slides with RNase A after hybridization were key steps in reducing the background signal.

Modifications to the procedure had to be made for each tissue and probe to optimize the signal strength and to reduce the background. During the pretreatment of tissues the endogenous peroxidase quenching step was increased from $1 \%$ hydrogen peroxide for kidney (and most tissues) to $3 \%$ for skin samples and fibrofolliculoma. Also, the optimal concentration of RNase A ranged from 0.1 to $1 \mu \mathrm{g} / \mathrm{ml}$ depending on the strength of the nonspecific signal from the sense probe and the binding strength of the antisense probe. For the Ker6IRS1 probe, the background for the sense probe was still high at an RNase concentration of $0.625 \mu \mathrm{g} / \mathrm{ml}$ necessitating an increase to $1.0 \mu \mathrm{g} / \mathrm{ml}$ to obtain a signal-free sense slide. However, at an RNase A concentration of $0.625 \mu \mathrm{g} /$ $\mathrm{ml}$ the $B H D$ signal for the antisense and sense probes was absent. The optimal RNase A concentration for the $B H D$ sense and antisense probes was 10 -fold lower at $0.131 \mu \mathrm{g} / \mathrm{ml}$.

\section{Expression of BHD mRNA in Skin and Skin Appendages}

A riboprobe from the Ker6IRS1 gene ${ }^{16}$ was used to optimize the in situ hybridization protocol and as a control to visualize the different regions of the hair follicle. The Ker6IRS1 riboprobe bound specifically to all three layers of the inner root sheath (Henle, 
Huxley and inner cuticle) and sense probe slides were void of signal (Figure 2a and b). This indicated that the in situ hybridization procedure was opti- mized for signal strength and produced a negative sense control. The $B H D$ riboprobe, designed from the $3^{\prime}$ end of the $B H D$ gene cDNA, hybridized to the
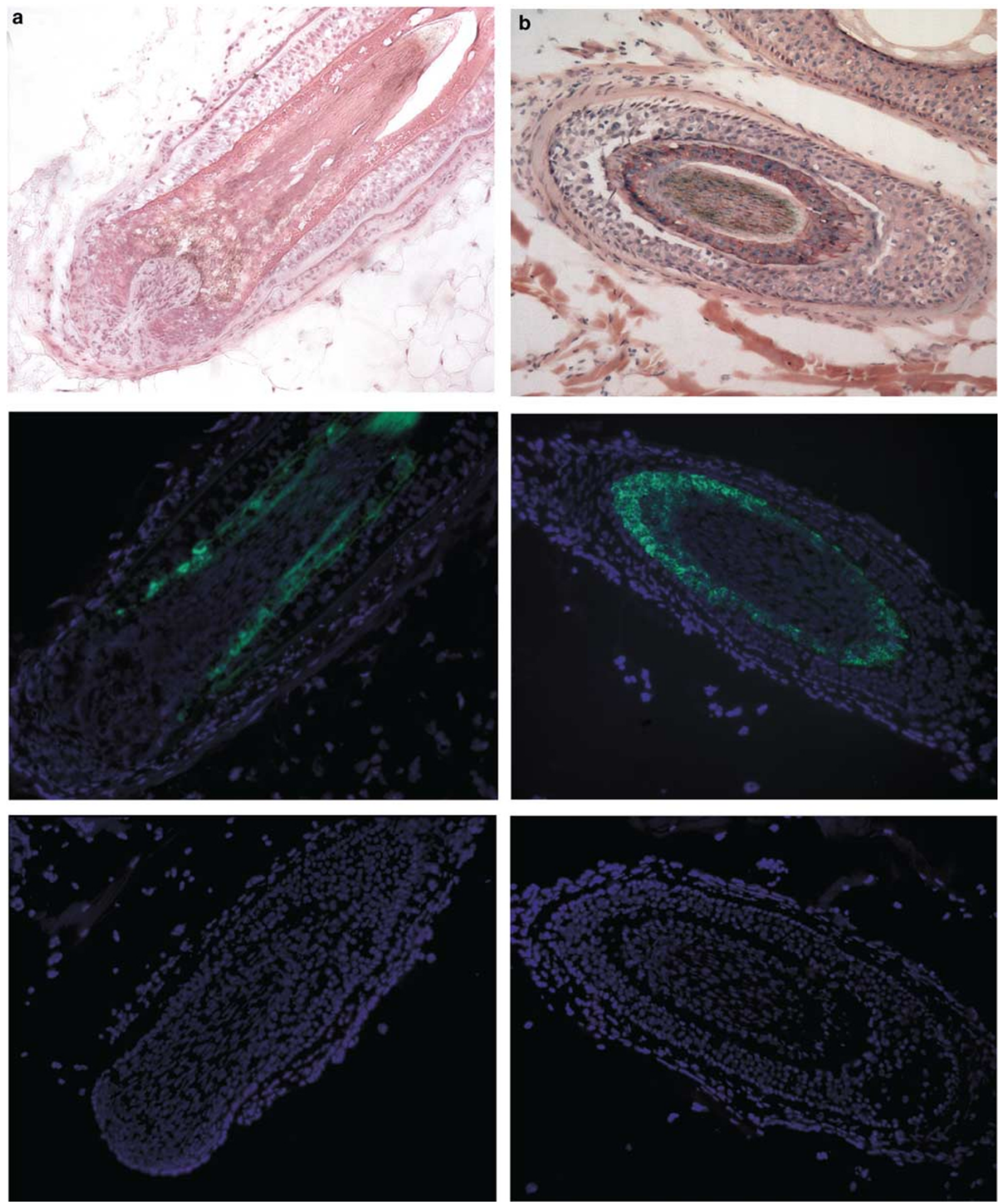

Figure 2 In situ hybridization of a riboprobe from Ker6IRS1 to a (a) longitudinal and (b) cross-section hair follicle from a human scalp sample demonstrated expression specific to the inner root sheath. Top panels, hematoxylin and eosin staining; middle panels, antisense probe (green); bottom panels, sense probe. Nuclei counterstained with DAPI (blue). Original magnification, $\times 100$. 
three layers of the inner root sheath as well as to the outer root sheath (Figure $3 \mathrm{a}$ and b). Both longitudinal and cross-sectioned hair follicles were hybridized and no signal was seen in the dermal papilla, the fibrous root sheath, the arrector pili muscle or the melanocytes. The sense probe control slides were void of signal (Figure 3 ).

The epidermis exhibited strong positive signal in the keratinocytes of the spinous layer, but a weaker signal in the basal cell layer directly above the dermis (Figure 4a). The stratum corneum and the granular layer of the epidermis did not show BHD mRNA signal. In the dermis, discrete expression was seen in a wide variety of cells within the collagen fibers including nerve cells, lymphocytes, macrophage, mast cells and fibroblasts (below the epidermis in Figure 4a). The collagen fibers themselves, as well as the blood vessels, did not fluoresce. The sebaceous glands branch from the outer root sheath of the hair follicle. The keratinocytes surrounding the sebaceous glands of the hair follicle and the eccrine glands (sweat glands) in the skin were
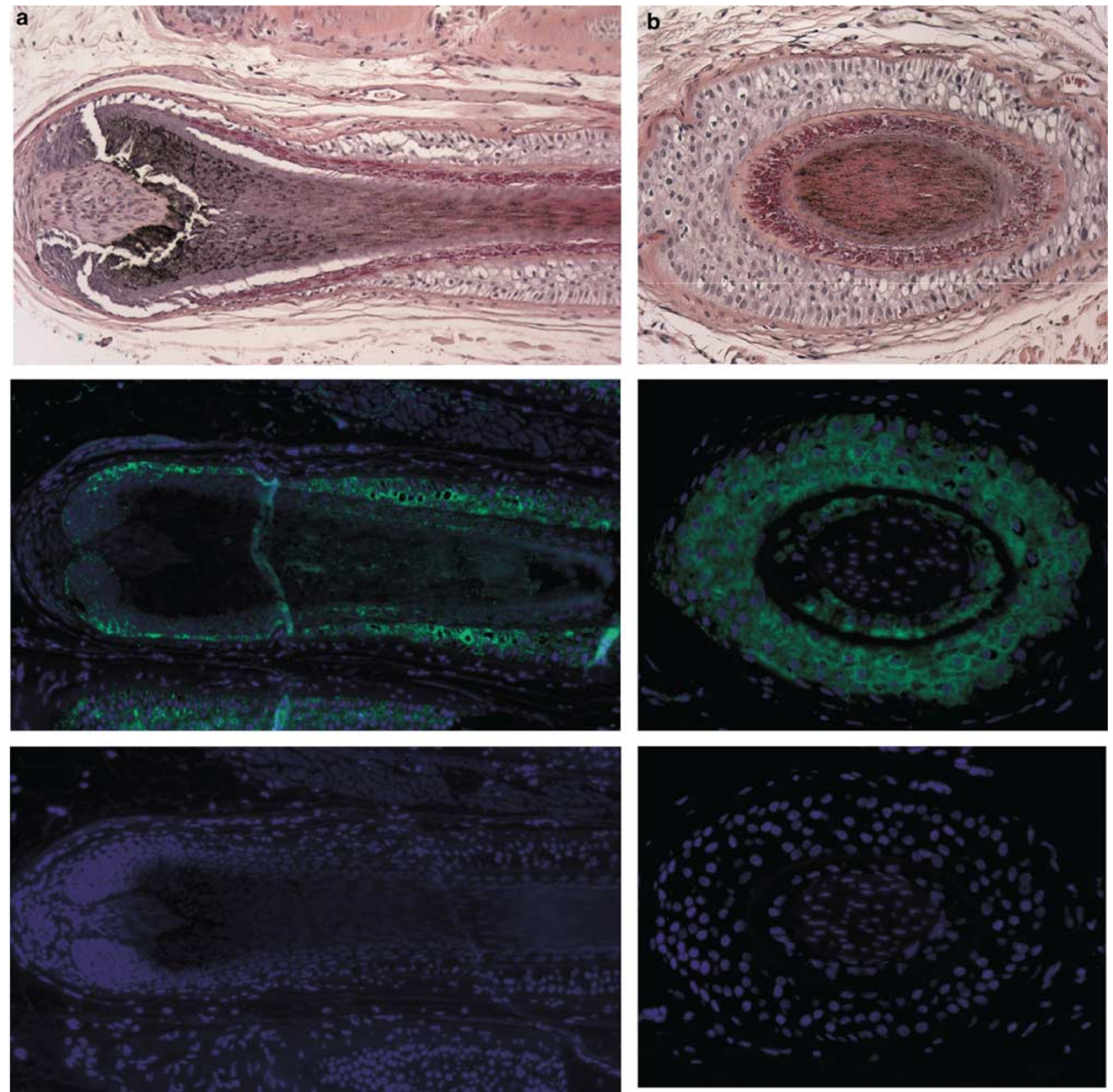

Figure 3 In situ hybridization of a riboprobe to BHD mRNA to the inner and outer root sheaths of a (a) longitudinal and (b) cross-section hair follicle from a human scalp sample. Top panels, hematoxylin and eosin staining; middle panels, antisense probe (green); bottom panels, sense probe. Nuclei counterstained with DAPI (blue). Original magnification, $\times 100$. 


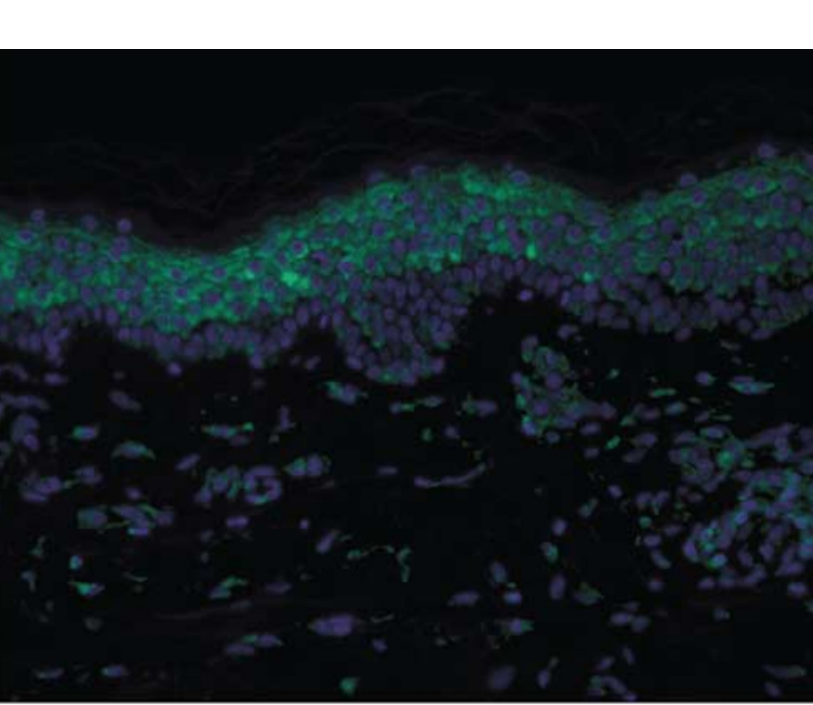

a
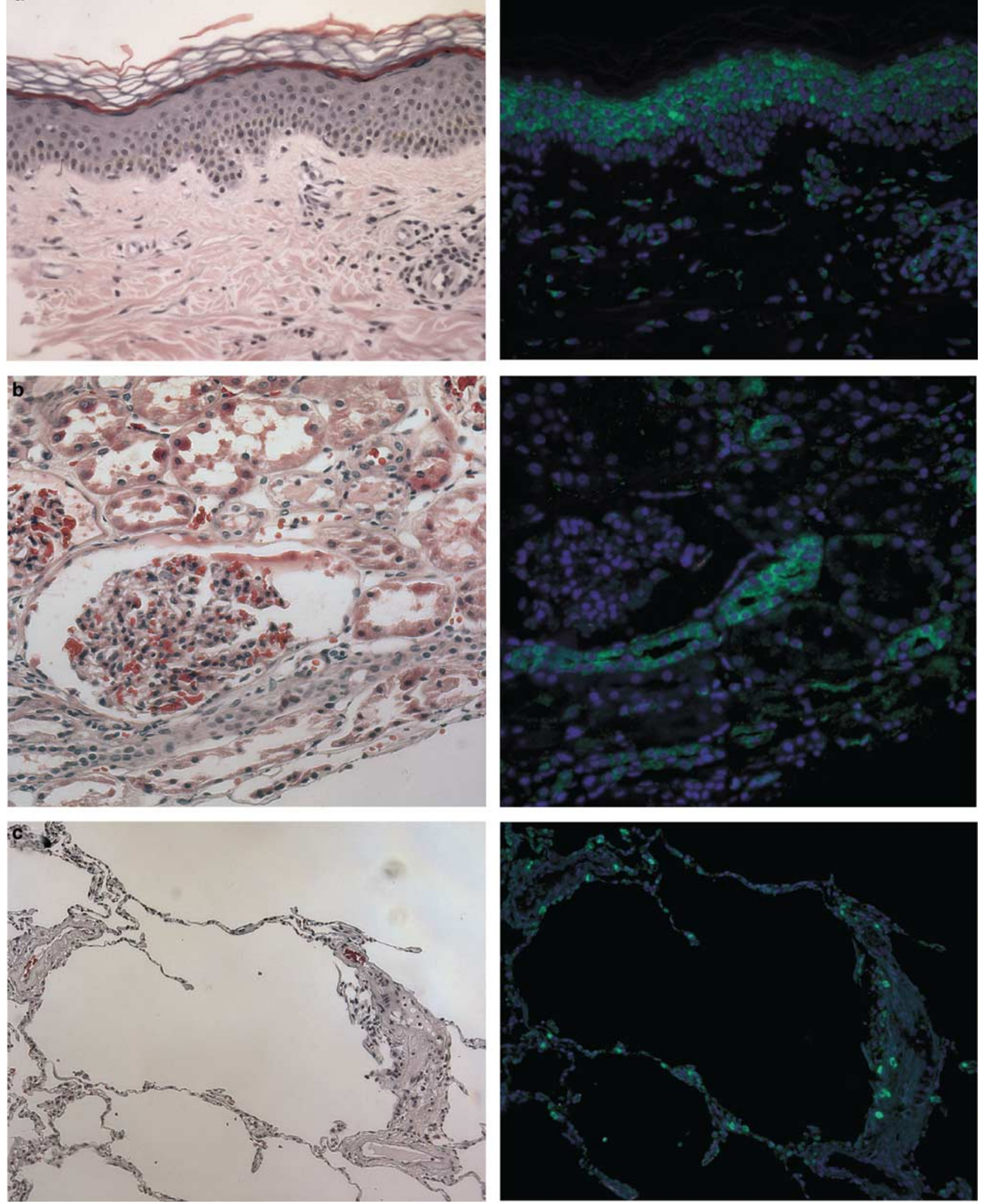

Figure 4 In situ hybridization of a BHD mRNA probe to tissues involved in the BHD phenotype: skin, kidney and lung. (a) Skin. BHD mRNA signal was highest in the spinous layer of the epidermis with expression in discrete cells of the dermis. (b) Kidney. BHD mRNA expression was seen in the distal nephron of the kidney. (c) Lung. BHD mRNA expression predominates in the stromal cells, but was also found in the type 1 pneumocytes. Left panels, hematoxylin and eosin staining; right panels, antisense probe. Nuclei counterstained with DAPI (blue). Original magnification, $\times 200$ for (a) and (b) and $\times 200$ for (c). 
positive for $B H D$ mRNA expression, but demonstrated less fluorescence than the hair follicle inner root sheath or outer root sheath (data not shown).

The expression pattern of $B H D$ mRNA in tissues associated with the BHD syndrome and other normal and neoplastic tissues is summarized in Table 1. All sense control slides for tissues were void of signal (data not shown).

\section{Expression of BHD mRNA in the Kidney and Lung, Tissues Involved in the BHD Phenotype}

In the kidney, in situ hybridization localized $B H D$ mRNA strongly to the distal nephron and collecting duct and showed a weak distribution of signal in the proximal tubules, but no signal in the glomeruli (Figure 4b).

The stromal cells (ie macrophage, fibroblasts, lymphocytes) within the connective tissue of the lung exhibited strong expression of $B H D$ mRNA, while the type 1 pneumocytes showed a weak signal throughout the lung tissue (Figure 4c). In addition, the macrophage within the spaces of the alveolar septa showed very strong $B H D$ expression (data not shown).

\section{BHD mRNA Expression in Exocrine Glands and in Other Normal Human Tissues}

Since the BHD protein, folliculin, is a novel protein with no known function, $B H D$ mRNA expression was evaluated on a normal tissue array in order to gain information about the role of folliculin in the cell based on the tissue expression pattern. BHD mRNA expression was seen in a wide variety of tissues including tonsils, lymph nodes, spleen, brain, breast, bladder, testis, prostate, ovary, myometrium, pancreas and parotid gland.

The serous (acinar) glands of the parotid gland highly expressed $B H D$ mRNA while the mucinous glands and intercalated ducts did not express $B H D$ (Figure 5a). The enzyme-secreting acinar cells of the pancreas fluoresced, but not the insulin-regulating islet cells (Figure 5b). In the cerebrum of the brain the neurons strongly expressed BHD mRNA, but the glial cells did not. Purkinje cells in the cerebellum bordering the granular layer strongly expressed $B H D$ mRNA (Figure 5c). The tonsils highly expressed $B H D$ mRNA in the germinal centers where lymphocytes proliferate and then progress out (Figure $5 \mathrm{~d}$ ). Signal from the lymphocytes was also seen in the white pulp of the spleen, but not in the red pulp where red blood cells are found (data not shown).

\section{Tissues with no BHD mRNA Expression}

No expression was seen in the heart, colon epithelium, mucinous glands of colon and parotid, adrenal gland, liver hepatocytes, stratified muscle, thyroid and in areas surrounding blood vessels (data not shown).

\section{BHD mRNA Expression Pattern in Fibrofolliculomas and Kidney Tumors from Patients Affected with BHD}

Fibrofolliculomas, the dermatologic skin lesions diagnostic for the BHD syndrome, were evaluated for expression with the $B H D$ riboprobe. Strong expression of $B H D$ mRNA was observed in the proliferating epithelial strands of fibrofolliculomas, as well as in keratinocytes within the spinous layer of the epidermis and surrounding the sebaceous glands (Figure 6a).

Several cases of renal tumors with chromophobe, clear cell and hybrid oncocytoma histology from patients with BHD were evaluated for $B H D$ mRNA expression; almost no expression of BHD mRNA was detected in these renal tumors relative to adjacent normal kidney parenchyma (Figure $6 \mathrm{~b}$ and $\mathrm{c}$ ). Lung blebs obtained from BHD patients who were treated for a spontaneous pneumothorax showed the same expression level of $B H D$ mRNA in stromal cells and type 1 pneumocytes as seen in the lung tissue of unaffected individuals (data not shown).

\section{BHD mRNA Expression in Neoplastic Tissues}

Neoplastic tissues on a Cancer Tissue Array were examined for $B H D$ mRNA expression and compared with adjacent normal tissue sections. Several types of breast carcinomas including infiltrating ductal adenocarcinoma and lobular carcinoma were examined. $B H D$ mRNA was highly expressed in these breast carcinomas (Figure 7a) as well as in normal breast tissue. In normal breast tissue, the epithelial mammary glands highly expressed $B H D$ mRNA with minor expression in the fibroblasts of the surrounding stroma (Figure $7 \mathrm{~b}$ ). Serous adenocarcinoma and cystadenocarcinoma of the ovary expressed $B H D$ at high levels, as did adjacent normal ovary tissue (data not shown). In adenocarcinoma of the prostate, the expression of the BHD mRNA in the epithelial cells was much higher than in the adjacent normal prostate tissue (Figure 7c). In normal tissue, the basal cells lining the prostate glands showed high expression, but only minor expression was seen in epithelial cells of the glands (Figure 7d).

A tissue array containing renal tumors of various histologic types was evaluated for $B H D$ expression. All five histologic variants seen in renal tumors of BHD patients including clear cell, papillary, oncocytoma, chromophobe and oncocytic hybrid, were represented. These tumor samples were obtained from patients with von Hippel-Lindau disease (clear cell), Hereditary Papillary Renal Carcinoma (papillary) or BHD (oncocytoma, chromophobe and oncocytic hybrid). All renal tumors showed severely reduced expression of $B H D$ regardless of histology; 

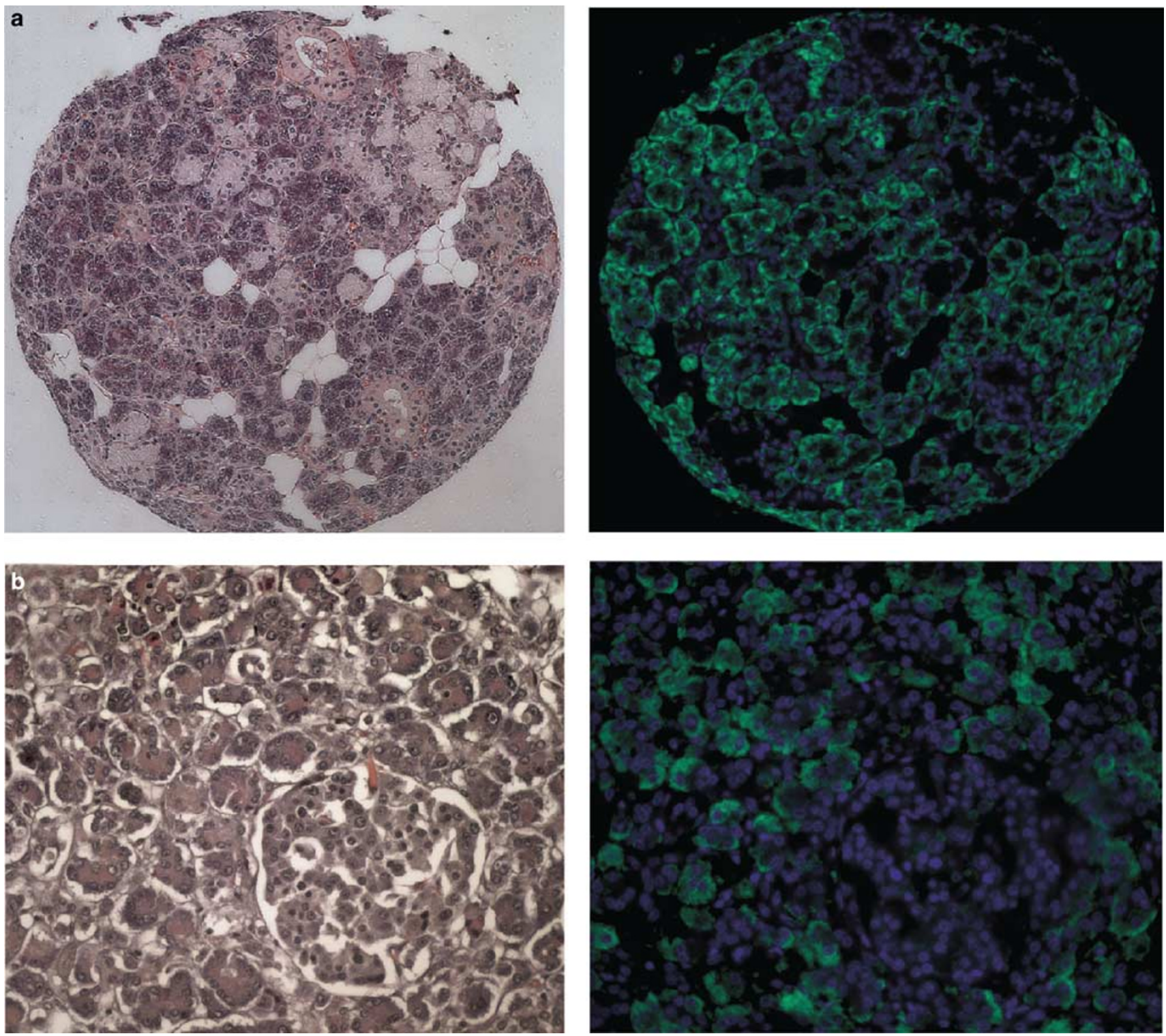

Figure 5 In situ hybridization of a BHD mRNA probe to (a) serous cells of parotid tissue, (b) acinar cells of pancreas tissue, (c) Purkinje cells in the cerebellum of the brain, and (d) lymphocytes in the germinal center of the tonsil. Left panels, hematoxylin and eosin staining; right panels, antisense probe. Nuclei counterstained with DAPI (blue). Original magnification, $\times 100$ for (a), (c) and (d), and $\times 200$ for (b).

in fact, papillary renal tumors did not show any $B H D$ expression (data not shown).

\section{Discussion}

In an effort to learn more about the biological function of the $B H D$ gene and its role in the skin, lung and kidney phenotype observed in patients with BHD syndrome, we evaluated expression of BHD mRNA in normal and neoplastic human tissues by fluorescent in situ hybridization. We found that BHD mRNA was widely, but not universally expressed in the organs and tissues examined with selective expression in certain cell types. For example, in the pancreas, $B H D$ mRNA was detected in the acinar cells, but not in the islets of Langerhans. In the kidney, $B H D$ mRNA was expressed strongly in the distal nephron, but only weakly in the proximal renal tubules, and was undetectable in glomeruli. BHD mRNA was expressed in keratinocytes of the spinous layer of the epidermis, but only weakly in cells of the basal layer of the epidermis. The expression of $B H D$ mRNA in certain cell types within each tissue may suggest that $B H D$ participates in particular cellular processes rather than serving as a housekeeping gene.

Notably, BHD mRNA was expressed in many diverse tissues and cell types such as the skin and its appendages, lymphocytes in the germinal centers of lymph nodes, neurons in the cerebrum and Purkinje cells in the cerebellum. This wide distribu- 

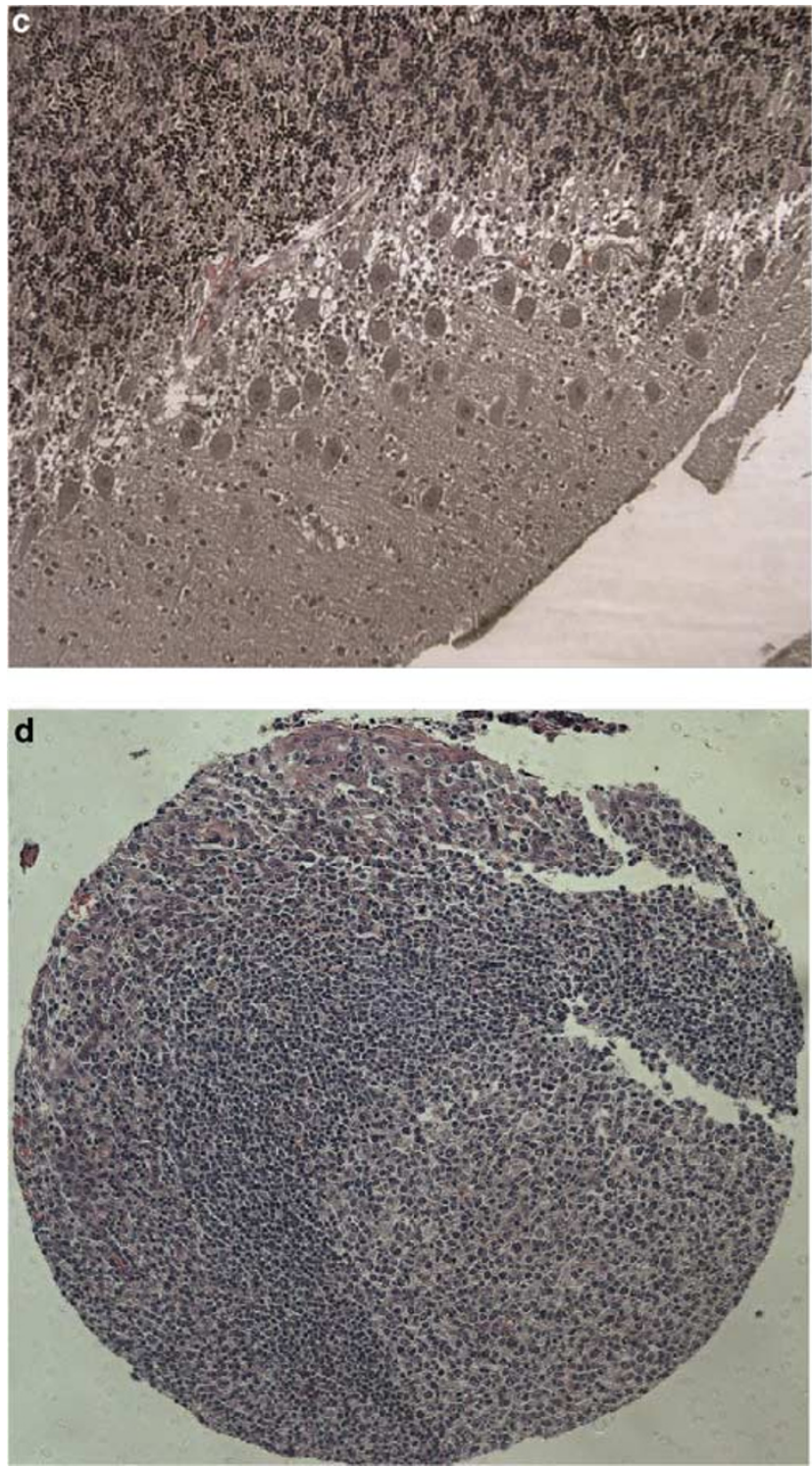

Figure 5 (Continued).

tion of $B H D$ mRNA expression suggests an important fundamental role for $B H D$ in biological processes within a variety of tissues.

The strong expression of $B H D$ in cell types with a secretory function, such as the acinar cells of the pancreas and parotid gland, may suggest a possible role of the BHD protein in secretion. Of interest is a report of a parotid oncocytoma in a BHD patient. ${ }^{17}$ Additionally, strong $B H D$ expression in ductal cells of the breast, collagen-producing fibroblasts, and antibody-producing lymphocytes lends support to a possible secretory function. Alternatively, $B H D$ mRNA expression in type I pneumocytes, which play a role in surfactant turnover by pinocytosis, ${ }^{18}$ and expression in alveolar macrophage, may suggest a role for BHD protein in endocytosis or phagocytosis. Experiments designed to test these and other
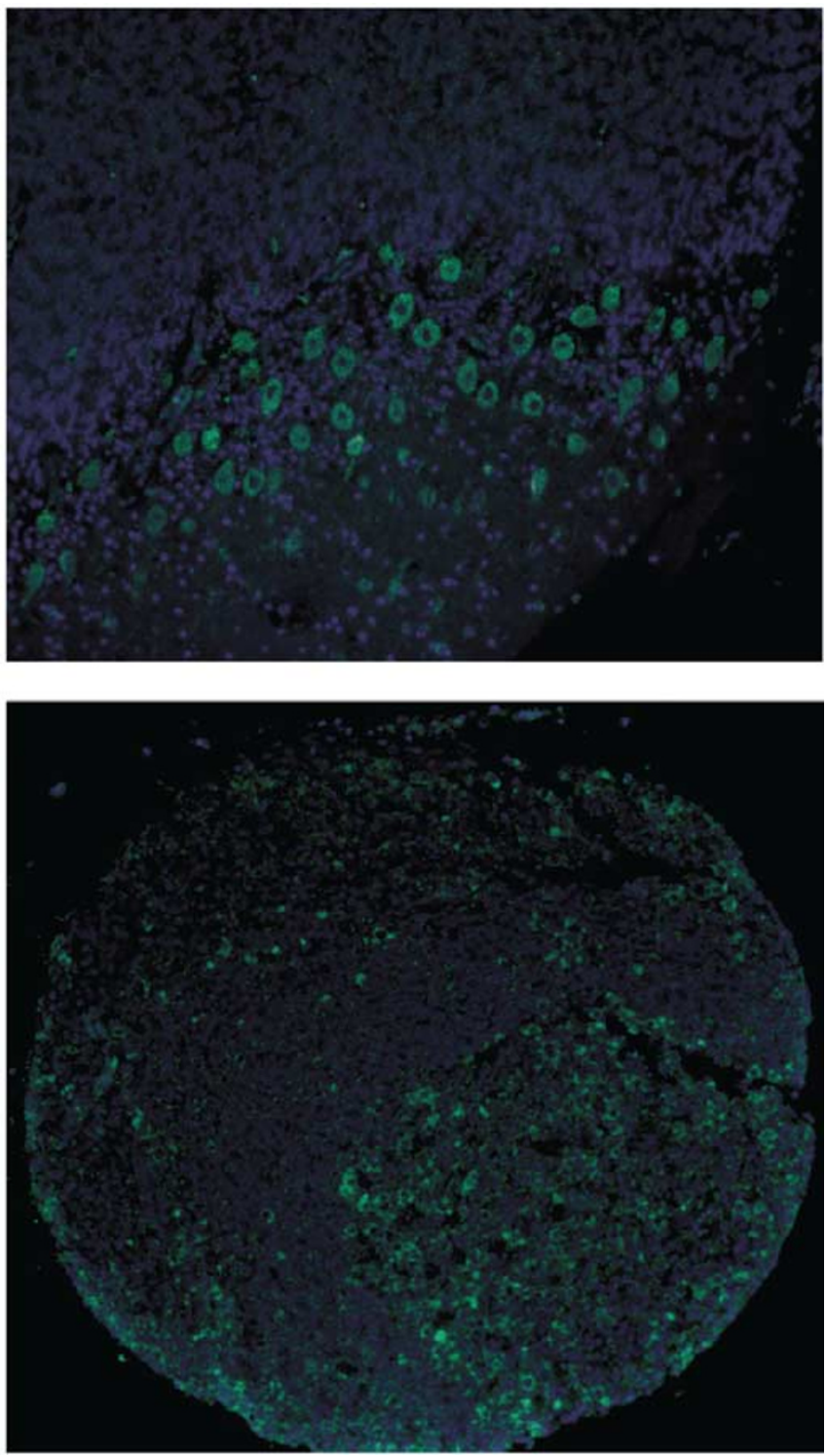

possible functions for the BHD protein in cellular processes are currently underway.

The primary clinical characteristics of the BHD syndrome are cutaneous skin lesions known as fibrofolliculomas, spontaneous pneumothorax, and a spectrum of histologic types of renal carcinoma. Examination of the tissues that are the targets of the disease showed that BHD mRNA was expressed strongly in the epithelial strands of the fibrofolliculomas, but not expressed or expressed weakly in renal tumors from patients affected with the BHD syndrome. Germline mutations in the BHD gene accompanied by somatic inactivation of the remaining $B H D$ allele, through $\mathrm{LOH}(20 \%)^{19}$ or somatic mutations $(50 \%$; C Vocke, manuscript in preparation), were identified in renal tumors from BHD patients. $\mathrm{LOH}$ on $17 \mathrm{p}$ has been reported in sporadic 
Table 1 BHD mRNA expression in normal and neoplastic tissues

\begin{tabular}{|c|c|c|c|}
\hline Normal tissue & Expression $^{\mathrm{a}}$ & Abnormal/neoplastic tissue & Expression $^{\mathrm{a}}$ \\
\hline Skin & & Fibrofolliculoma & \\
\hline Inner root sheath of hair follicle & +++ & Epithelial strands of fibrofolliculoma & +++ \\
\hline Outer root sheath of hair follicle & +++ & Mucin-rich stroma & - \\
\hline Dermal papilla of hair follicle & - & Collagen bundles & - \\
\hline Fibrous root sheath of hair follicle & - & & \\
\hline Matrix cells of hair follicle & - & & \\
\hline Keratinocytes around sebaceous gland & +++ & & \\
\hline Eccrine (sweat) glands & +++ & & \\
\hline Spinous layer keratinocytes of epidermis & +++ & & \\
\hline Basal cell layer of epidermis & \pm & & \\
\hline $\begin{array}{l}\text { Discrete cells in dermis such as fibroblasts, } \\
\text { macrophage and nerve cells }\end{array}$ & ++ & & \\
\hline Collagen fibrils of dermis & - & & \\
\hline Kidney & & Renal neoplasms & \\
\hline Distal tubules and collecting duct & +++ & Renal oncocytoma & \pm \\
\hline Proximal tubules & \pm & Clear cell renal cancer & \pm \\
\hline \multirow[t]{3}{*}{ Glomeruli } & $\overline{-}$ & Chromophobe renal cancer & $\overline{ \pm}$ \\
\hline & & Papillary renal cancer & - \\
\hline & & Oncocytic hybrid & \pm \\
\hline Lung & & Lung bleb ${ }^{\mathrm{b}}$ & \\
\hline Type 1 pneumocytes & ++ & Type 1 pneumocytes & ++ \\
\hline $\begin{array}{l}\text { Stromal cells including fibroblasts and } \\
\text { macrophage }\end{array}$ & +++ & $\begin{array}{l}\text { Stromal cells including fibroblasts and } \\
\text { macrophage }\end{array}$ & +++ \\
\hline Parotid & & NA & \\
\hline Acinar cells of the serous gland & +++ & & \\
\hline Intercalated ducts & - & & \\
\hline Mucinous glands & - & & \\
\hline Pancreas & & NA & \\
\hline Acinar cells & +++ & & \\
\hline Islet cells & - & & \\
\hline Breast & & Breast carcinomas & \\
\hline \multirow[t]{5}{*}{ Acinar cells of epithelial ducts } & +++ & Adenocarcinoma & +++ \\
\hline & & Lobular carcinoma & +++ \\
\hline & & Ductal carcinoma & +++ \\
\hline & & Mucinous adenocarcinoma & +++ \\
\hline & & Infiltrating ductal adenocarcinoma & +++ \\
\hline Prostate & & Prostate adenocarcinoma & \\
\hline Basal cells of the prostate glands & +++ & Epithelial cells & +++ \\
\hline Epithelial cells of the prostate gland & \pm & & \\
\hline \multicolumn{4}{|l|}{ Tonsil } \\
\hline Germinal center & +++ & NA & \\
\hline Spleen & & NA & \\
\hline White pulp & +++ & & \\
\hline Red pulp & - & & \\
\hline Brain 1 & & NA & \\
\hline Neurons of the cerebrum & +++ & & \\
\hline Glial cells of the cerebrum & - & & \\
\hline \multirow{2}{*}{\multicolumn{4}{|c|}{ Bladder }} \\
\hline & & & \\
\hline Transitional epithelial & +++ & NA & \\
\hline Ovary & & Ovarian cancer & \\
\hline \multirow[t]{3}{*}{ Mesenchymal cells } & +++ & Serous adenocarcinoma & +++ \\
\hline & & Cystadenocarcinoma & +++ \\
\hline & & Mucinous cystadenocarcinoma & +++ \\
\hline \multicolumn{4}{|c|}{ 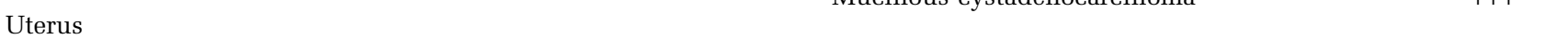 } \\
\hline Myometrium & +++ & NA & \\
\hline Colon & - & NA & \\
\hline Adrenal gland & - & NA & \\
\hline Liver & - & NA & \\
\hline Stratified muscle & - & NA & \\
\hline Heart & - & NA & \\
\hline Thyroid & - & NA & \\
\hline
\end{tabular}

aExpression: +++ strong expression; ++ moderate expression; \pm low expression; - no expression.

${ }^{b}$ Lung surgical sample removed from BHD patient with pneumothorax.

NA: not available. 

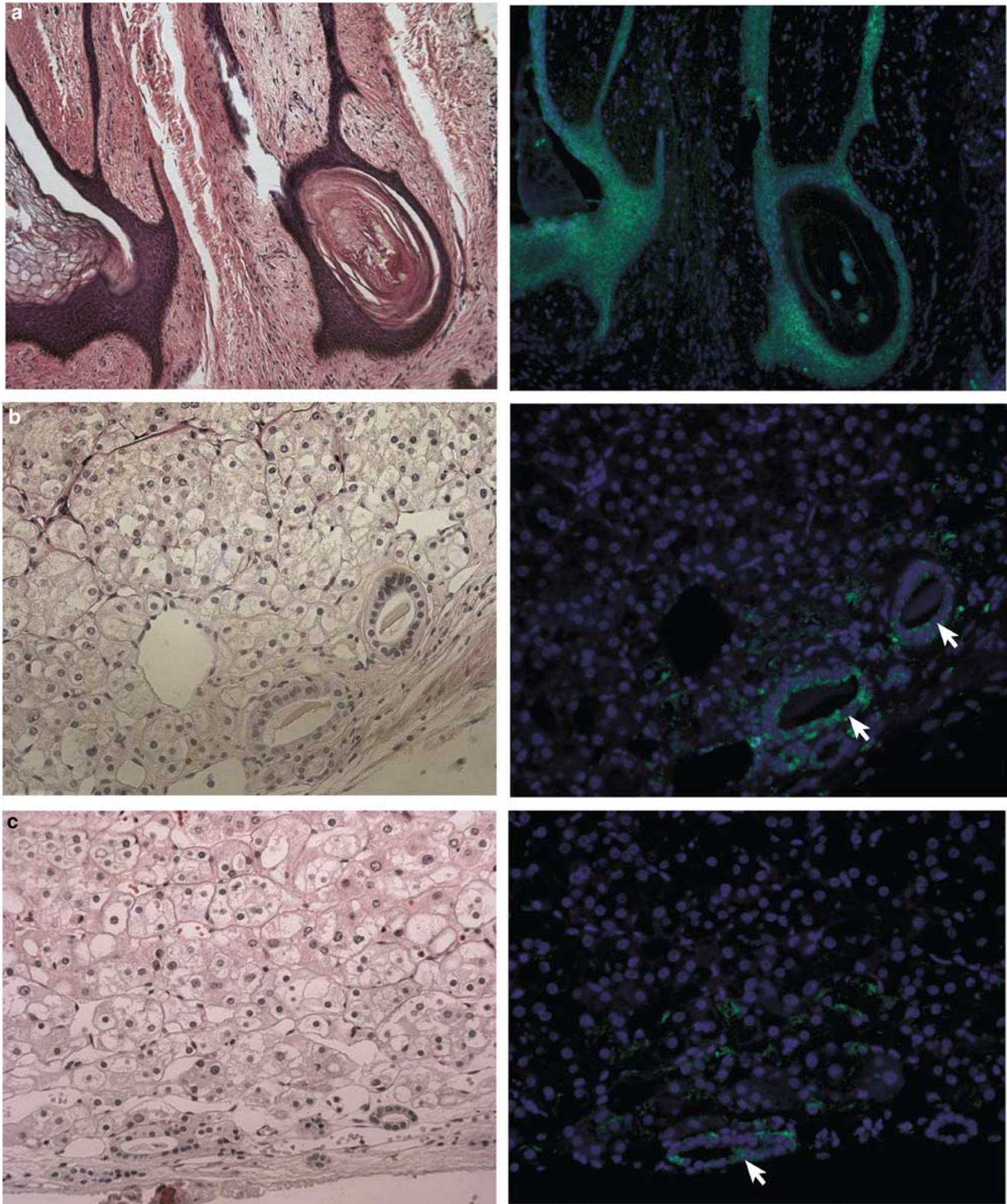

Figure 6 In situ hybridization of a BHD mRNA probe to sections from a (a) fibrofolliculoma, (b) chromophobe renal tumor, and a (c) oncocytic hybrid renal tumor. Note the BHD mRNA expression in normal tissue bordering the renal tumor sections (arrows). Left panels, hematoxylin and eosin staining; right panels, antisense probe. Nuclei counterstained with DAPI (blue). Original magnification, $\times 100$ for (a), and $\times 200$ for (b) and (c). 

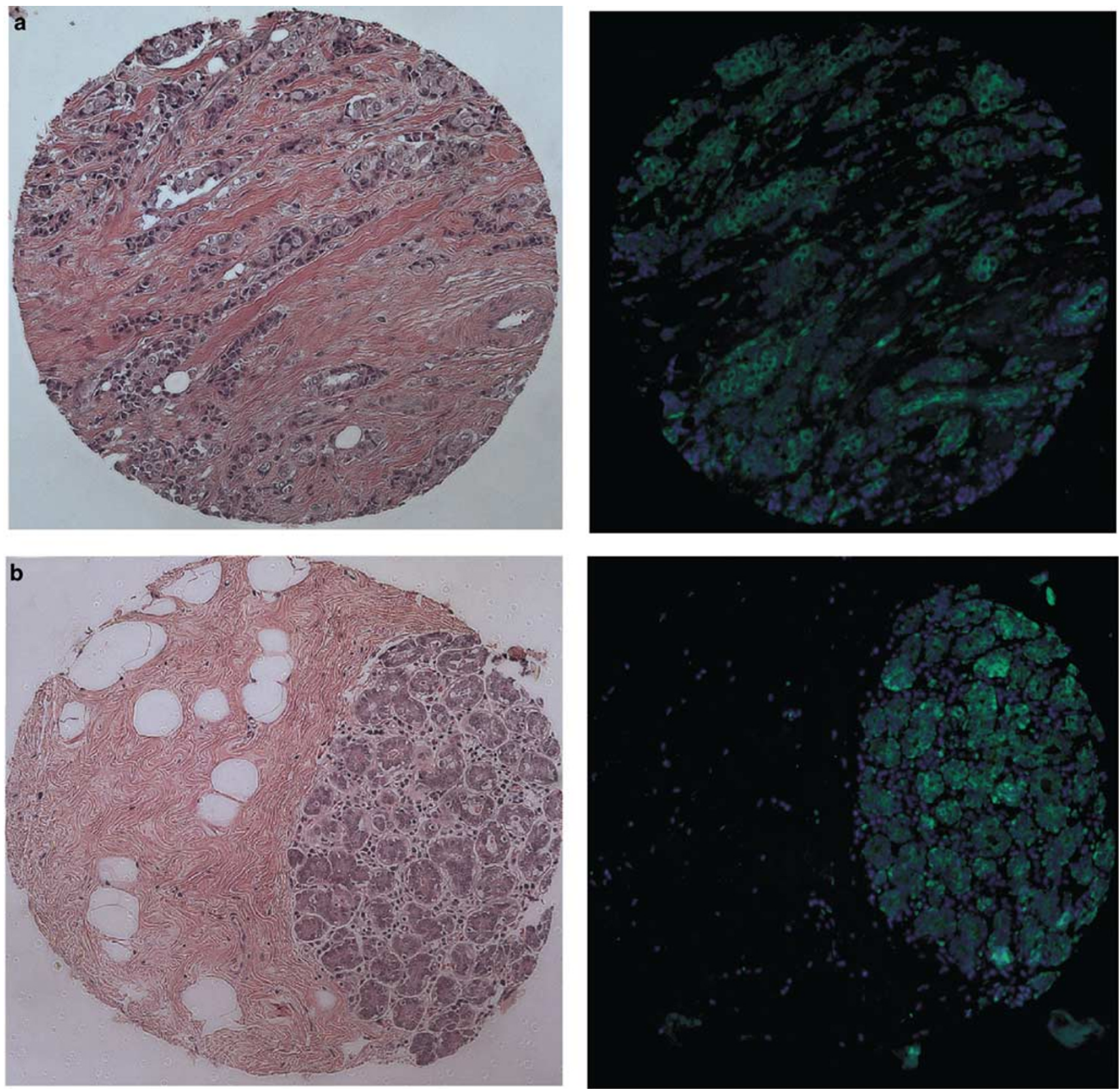

Figure 7 In situ hybridization of a BHD mRNA probe to sections from (a) ductal carcinoma of the breast, (b) normal breast tissue, (c) adenocarcinoma of the prostate, and (d) normal prostate tissue. Left panels, hematoxylin and eosin staining; right panels, antisense probe. Nuclei counterstained with DAPI (blue). Original magnification, $\times 100$.

renal tumors,$^{20}$ with one case also carrying a somatic BHD gene mutation. ${ }^{21}$ These observations suggest a tumor suppressor role for $B H D$, which is further supported by the reduced expression of the $B H D$ transcript in BHD renal tumors.

Studies are ongoing to evaluate tissue expression of the BHD protein, folliculin, using polyclonal antibodies prepared against synthetic peptides. The results of these studies will contribute further to our understanding of the role of the $B H D$ gene product, folliculin, in regulating cell proliferation and growth in skin, lung and kidney.

\section{Acknowledgements}

We thank Drs Jorge Toro, George Cotsarelis and Paul Duray for helpful discussions and Dr Steven Hewitt for providing the kidney tumor tissue array. This publication has been funded in part with Federal funds from the National Cancer Institute, National Institutes of Health, under Contract No. N01-C012400. The content of this publication does not necessarily reflect the views or policies of the Department of Health and Human Services, nor does mention of trade names, commercial products, 

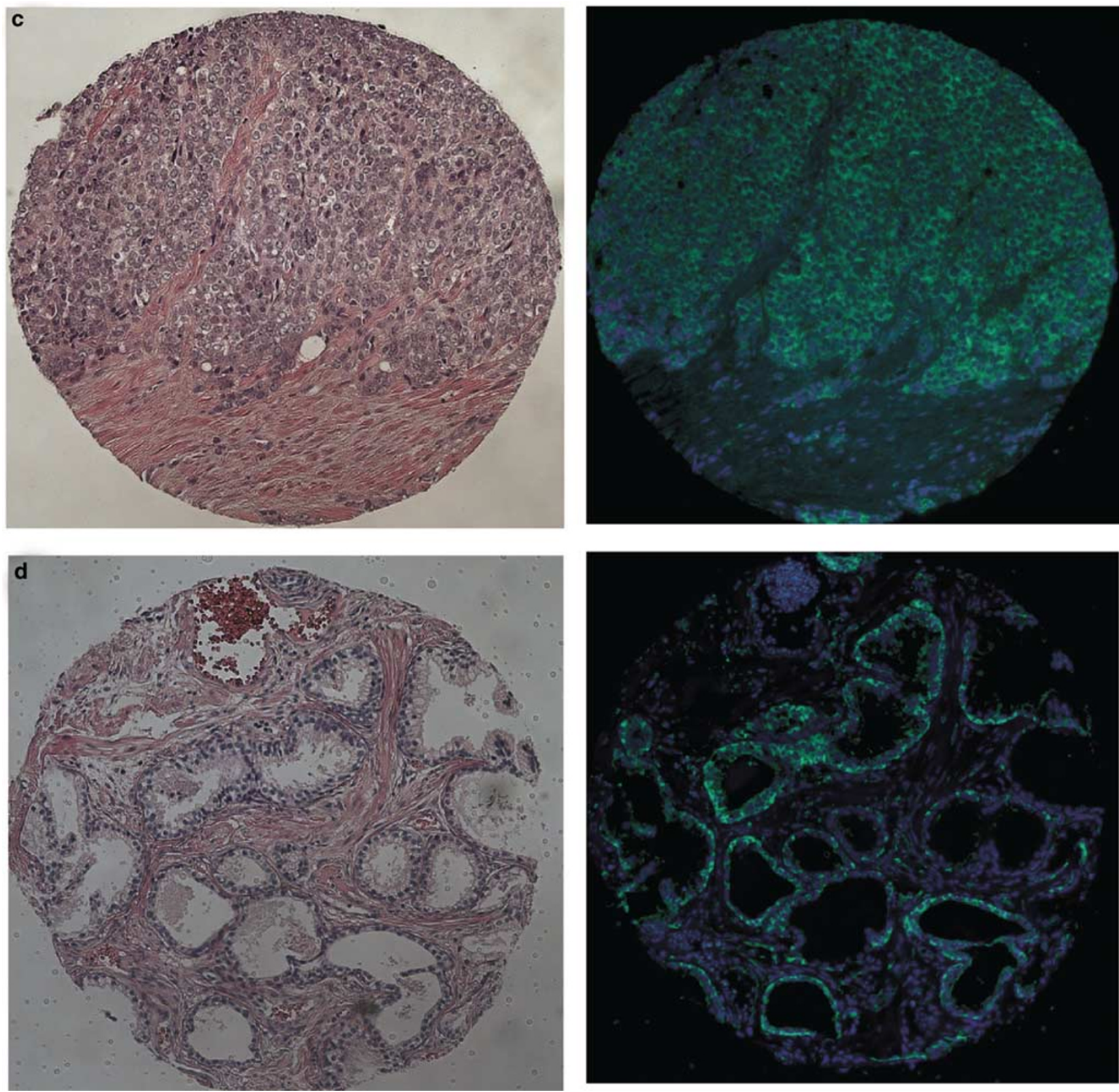

Figure 7 (Continued).

or organizations imply endorsement by the US Government. Research reported in this manuscript was part of a Master's degree thesis for the Graduate School of Hood College in Frederick, Maryland (MBW).

\section{References}

1 Birt AR, Hogg GR, Dube WJ. Hereditary multiple fibrofolliculomas with trichodiscomas and acrochordons. Arch Dermatol 1977;113:1674-1677.
2 Roth JS, Rabinowitz AD, Benson M, et al. Bilateral renal cell carcinoma in the Birt-Hogg-Dube syndrome. J Am Acad Dermatol 1993;29:1055-1056.

3 Toro JR, Glenn G, Duray P, et al. Birt-Hogg-Dube syndrome: a novel marker of kidney neoplasia. Arch Dermatol 1999;135:1195-1202.

4 Binet O, Robin J, Vicart M, et al. Fibromes perifolliculaires polypose colique familaile pneumothorax spontanes familiaux. Ann Dermatol Venereol 1986;113: 928-930.

5 Zbar B, Alvord WG, Glenn G, et al. Risk of renal and colonic neoplasms and spontaneous pneumothorax in the Birt-Hogg-Dube syndrome. Cancer Epidemiol Biomarkers Prev 2002;11:393-400. 
6 Pavlovich CP, Walther MM, Eyler RA, et al. Renal tumors in the Birt-Hogg-Dube syndrome. Am J Surg Pathol 2002;26:1542-1552.

7 Tickoo SK, Reuter VE, Amin MB, et al. Renal oncocytosis: a morphologic study of fourteen cases. Am J Surg Pathol 1999;23:1094-1101.

8 Schmidt LS, Warren MB, Nickerson ML, et al. BirtHogg-Dube syndrome, a genodermatosis associated with spontaneous pneumothorax and kidney neoplasia, maps to chromosome 17p11.2. Am J Hum Genet 2001;69:876-882.

9 Khoo SK, Bradley M, Wong FK, et al. Birt-Hogg-Dube syndrome: mapping of a novel hereditary neoplasia gene to chromosome 17p12-q11.2. Oncogene 2001;20: 5239-5242.

10 Nickerson ML, Warren MB, Toro JR, et al. Mutations in a novel gene lead to kidney tumors, lung wall defects, and benign tumors of the hair follicle in patients with the Birt-Hogg-Dube syndrome. Cancer Cell 2002;2: 157-164.

11 Lium B, Moe L. Hereditary multifocal renal cystadenocarcinomas and nodular dermatofibrosis in the German shepherd dog: macroscopic and histopathologic changes. Vet Pathol 1985;22:447-455.

12 Jonasdottir TJ, Mellersh CS, Moe L, et al. Genetic mapping of a naturally occurring hereditary renal cancer syndrome in dogs. Proc Natl Acad Sci USA 2000;97:4132-4137.

13 Lingaas F, Comstock KE, Kirkness EF, et al. A mutation in the canine BHD gene is associated with hereditary multifocal renal cystadenocarcinoma and nodular dermatofibrosis in the German Shepherd dog. Hum Mol Genet 2003;12:3043-3053.

14 Hino O, Okimoto $\mathrm{K}$, Kouchi $\mathrm{M}$, et al. A novel renal carcinoma predisposing gene of the Nihon rat maps on chromosome 10. Jpn J Cancer Res 2001;92:1147-1149.

15 Okimoto K, Kouchi M, Kikawa E, et al. A novel 'Nihon' rat model of a Mendelian dominantly inherited renal cell carcinoma. Jpn J Cancer Res 2000;91:1096-1099.

16 Langbein L, Rogers MA, Praetzel S, et al. A novel epithelial keratin, hK6irs1, is expressed differentially in all layers of the inner root sheath, including specialized Huxley cells (Flugelzellen) of the human hair follicle. J Invest Dermatol 2002;118:789-799.

17 Liu V, Kwan T, Page EH. Parotid oncocytoma in the Birt-Hogg-Dube syndrome. J Am Acad Dermatol 2000; 43:1120-1122.

18 Junqueira LC, Carneiro J, Kelly R. The respiratory system. In: Barnes D (ed). Basic Histology, 9th edn. Appleton and Lange: Stamford, CT, 1998, pp 327-346.

19 Pavlovich CP, Schmidt LS, Warren MB, et al. Genetic analysis of renal cell carcinomas in Birt-Hogg-Dube syndrome. In: Proceedings of the American Association for Cancer Research, April 6-10, San Francisco, CA, 2002.

20 Khoo SK, Kahnoski K, Sugimura J, et al. Inactivation of BHD in sporadic renal tumors. Cancer Res 2003;63: 4583-4587.

21 da Silva NF, Gentle D, Hesson LB, et al. Analysis of the Birt-Hogg-Dube (BHD) tumour suppressor gene in sporadic renal cell carcinoma and colorectal cancer. J Med Genet 2003;40:820-824. 